\title{
Home-based rehabilitation: Physiotherapy student and client perspectives
}

\author{
D Parris, ${ }^{1}$ BSc (Physio), MPhil (HSE); S C van Schalkwyk, ${ }^{2}$ PhD; D V Ernstzen, ${ }^{1}$ BSc (Physio), MPhil (Higher Ed)
}

${ }^{1}$ Division of Physiotherapy, Department of Interdisciplinary Health Sciences, Faculty of Medicine and Health Sciences, Stellenbosch University, Cape Town, South Africa

${ }^{2}$ Centre for Health Professions Education, Faculty of Medicine and Health Sciences, Stellenbosch University, Cape Town, South Africa

Corresponding author: D Parris (dparris@sun.ac.za)

Background. Home-based rehabilitation (HBR) in under-resourced areas in a primary healthcare (PHC) context exposes students to the real-life situations of their clients. There is a scarcity of literature on student and client experiences of HBR in the physiotherapy context. Increased knowledge of HBR could result in an enhanced experience for both student and client. This study sought to discover the perceptions of final-year physiotherapy students and their clients relating to their experiences of HBR during a PHC placement in a resource-constrained setting.

Objectives. To explore the experiences and perceptions of physiotherapy students and their clients regarding HBR as part of clinical training in resource-constrained settings. To discover the barriers to and facilitators of effective HBR.

Methods. An exploratory case study was performed. A qualitative phenomenological research design in the interpretivist paradigm was used. Semistructured interviews were conducted with clients $(n=7)$ living in an under-resourced setting, who had received HBR from physiotherapy students. Paired interviews were conducted with final-year physiotherapy students $(n=6)$ after their HBR placement.

Results. Clients appreciated the students' services; however, data revealed communication barriers and unmet expectations. Students reported struggling to adapt to the context, resulting in interventions not being sufficiently client-centred. They voiced a need for language competency and earlier exposure to such contexts.

Conclusion. Exposure to real-life situations in under-resourced settings in HBR provides valuable situated and authentic learning opportunities for physiotherapy students. The experience can be useful in preparing graduates to address the needs of the populations they serve during community service.

Afr J Health Professions Educ 2016;8(1):59-64. DOI:10.7196/AJHPE.2016.v8i1.561

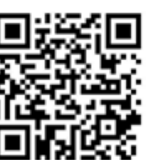

South Africa (SA)'s health system places the focus on primary healthcare (PHC) ${ }^{[1]}$ which deals with the health needs of a population in the communities where they live. ${ }^{[1]} \mathrm{PHC}$ policy incorporates community-based rehabilitation as part of continuity of care and includes interventions in a client's home. ${ }^{[1]}$ Physiotherapy education should produce graduates who are competent in addressing the health needs of the people within the community context. ${ }^{[2]}$ Graduates need to serve as health advocates and be accountable for making informed decisions to improve healthcare. ${ }^{[3]}$ To enable them to be more effective in this regard, graduates need exposure to the realities of the healthcare system, socioeconomic health determinants, and clients' real-life situations through PHC.

To train students effectively, authentic learning opportunities are necessary in the communities they will ultimately serve, particularly during their compulsory community service year. ${ }^{[2,4]}$ These communities are often situated in under-resourced areas. Experiencing the real-life context of clients is vital for students so that they will ultimately provide effective interventions and develop social responsibility. ${ }^{[2]}$ Students witness the roles of poverty and society in health first-hand during home-based rehabilitation (HBR). Students report feeling overwhelmed when witnessing the realities of life in poor households. ${ }^{[4-6]}$

Factors such as diverse socioeconomic, racial, language and cultural backgrounds may affect healthcare interventions. Developing cultural competency, i.e. the ability to treat people from a culture different to one's own with respect and as equals, has become critical in physiotherapy training. ${ }^{[7]}$ Culturally competent and effective client-centred communication leads to improved client satisfaction, outcomes and compliance. ${ }^{[8]}$
Different skills and clinical reasoning processes are required for physiotherapeutic rehabilitation in a home context compared with clinic- or hospitalbased interventions. Tasker et al. ${ }^{[9]}$ report that during HBR, clinical reasoning should primarily consider the client and family needs within the home setting. Students need to be able to adjust the goal and process of the intervention to ensure efficacy and relevance to the clients' context. ${ }^{[7]}$ Listening attentively to clients can increase the students' understanding of all factors affecting clients' health, goals and quality of life and thereby improve client satisfaction. ${ }^{[8]}$ Other skills students may develop in this context include: increased insight, coping with complexity, ability to think on one's feet, assertiveness, building rapport, empowering others, enhanced observation skills, functioning in someone else's space, consideration of quality of life and function as applicable to the client, knowing when to discontinue treatment, integration of services, and ability to function as a professional with limited resources. ${ }^{[4,9,10]}$

The theory of situated learning, i.e. learning through active participation within a community of practice, underpins the educational experience of HBR. ${ }^{[1]}$ Authentic exposure in a client's home environment can result in experiential learning, thereby promoting transformative learning. ${ }^{[1]}$ Transformative learning, i.e. learning that changes one's view of the world, is a desired outcome of health professional education. ${ }^{[12]}$ HBR leads not only to academic learning and personal development, but also to an understanding of social accountability and responsibility. ${ }^{[2,3]}$ However, to gain maximum benefit from the learning opportunities available, students should be prepared effectively before exposure to HBR. ${ }^{[4]}$

There is a scarcity of literature on HBR in the physiotherapy context, specifically with regard to the students' or clients' perceptions. This study sought to discover the perceptions of final-year physiotherapy students 
and their clients regarding their experiences of HBR during a PHC clinical training placement in resource-constrained and diverse settings. Increased knowledge of HBR could improve the preparation of students for the placement, resulting in an enhanced experience for both students and clients.

\section{Method}

\section{Research design}

An exploratory case study was conducted, focusing on the phenomenon of HBR in an educational context. The phenomenological enquiry allowed an understanding of the real-life experiences and feelings of the participants. ${ }^{[13]}$ In-depth interviews generated qualitative data in an interpretivist paradigm, taking the clients' and students' perceptions as their reality. ${ }^{[14]}$

\section{Research context}

At Stellenbosch University (SU), Cape Town, SA, final-year physiotherapy students each spend 6 weeks at a community site learning to integrate and apply the principles of PHC and communitybased rehabilitation. Approximately 5 - 10 clients are seen in their homes per week. One of the assessed outcomes of this placement is that the students will be able to effectively evaluate and treat clients in their own homes. Before this placement, the students mostly treated clients in community health centres or hospital settings, which are more structured and better-equipped environments. Students receive 1 hour of supervision per week from their clinical supervisor; therefore, they mostly conduct HBR on their own. They have the assistance of a community member as a chaperone and translator one afternoon per week.

The research was conducted at a community placement site in an under-resourced community in the Western Cape, SA. The majority of residents are isiXhosa speaking and live in informal housing with minimal resources. ${ }^{[15]}$ Students provide the only physiotherapy service in the area. The clients receive HBR in their homes, as they are unable to access or afford transport to the nearest physiotherapy department.

\section{Participants}

Purposive sampling was used to invite participants who could share their experiences of HBR. ${ }^{[14]}$ Final-year physiotherapy students from SU were invited to participate in the study after they had completed their rotation in the area. Clients who had received treatment in their homes by these students were approached to participate.

\section{Data collection}

Single, face-to-face, semistructured interviews with individual clients were conducted in their homes. The student interviews comprised a reflective conversation between pairs of students who had conducted HBR together, as required by SU safety guidelines. ${ }^{[14]}$ The interviews were conducted in the home language (English, Afrikaans or isiXhosa) of each participant to enable more fluent answers and were recorded with their permission. Open-ended questions regarding their perceptions and experiences of HBR were used to stimulate conversation. The participants were asked about their perceptions about the barriers to and facilitators of effective HBR. Obtaining both groups' perceptions assisted in data triangulation. ${ }^{[16]}$ Students were also asked for their perspectives on the preparation for PHC and HBR. To minimise potential bias, the interviews were conducted by trained research assistants who were not associated with the SU Division of Physiotherapy.

\section{Data analysis}

The isiXhosa interviews with clients were independently translated into English and transcribed. To improve rigour, the transcriptions were checked for accuracy by an isiXhosa-speaking assistant. Member checking by clients was not feasible owing to low literacy levels. The researcher - the students' clinical supervisor - checked the transcriptions of the students' interviews. The data were subjected to inductive thematic analysis by the researcher. ${ }^{[14]}$ Codes were manually assigned to themes identified in the data and categorised accordingly. Iterative data analysis occurred to increase the dependability of the study ${ }^{[16]}$ To ensure credibility, member checking of a transcript and data coding by a student assisted in data verification. ${ }^{[16]}$ The researcher acknowledged that her role as an instrument in the study may have affected student data. To minimise this, she made use of peer debriefing with the co-authors at different points in the study to enhance the confirmability and trustworthiness of the data. ${ }^{[16]}$

\section{Ethical considerations}

Approval for the study was obtained from the SU Health Research Ethics Committee (S13/10/180) and the Western Cape Department of Health (RP032/2014). Signed informed consent was obtained from all participants. Participation was entirely voluntary and did not affect services to clients or influence student assessment. The interviews were conducted after the students' examination papers had been marked and feedback given in order to minimise any potential bias. Confidentiality was maintained during the process, with no identifying particulars of individual clients or students being kept.

\section{Results \\ Participants}

Seven clients, of a potential 12, were interviewed. Three clients were excluded owing to their inability to converse, 1 client was not traceable and 1 had died. All 6 students who had completed their HBR placement at the time of the study participated. The students and clients had different socioeconomic, racial, cultural and language backgrounds.

The data obtained from the interviews were analysed to identify themes and categories to promote understanding of HBR. The major themes and associated categories emerging from the client and student interviews are presented separately with supporting quotations.

\section{Client perspectives}

The major themes of appreciation and clientcentredness emerged from the client interviews. The main theme related to their appreciation of the treatment received, being treated in their home and attention paid to their goals (Table 1).

Categories under the theme of clientcentredness (Table 2) related to communication,

Table 1. Clients' appreciation of students

\begin{tabular}{ll}
\hline Category & Supporting quotations \\
\hline Impact of treatment & $\begin{array}{l}\text { 'There is a difference since; I now am able to do things independently' (CL2) } \\
\text { 'Since they came, I can do so many things that I was not able to do before.' (CL2) }\end{array}$ \\
Treatment at home & $\begin{array}{l}\text { 'I appreciate the fact that students come to the house for therapy; it's expensive } \\
\text { to hire a car. Due to the location of my house it's too difficult for the car to get } \\
\text { there.' (CL1) }\end{array}$ \\
'Theatment goals & $\begin{array}{l}\text { 'I do want them to give me the exercises as is supposed to be. (CL2) } \\
\end{array}$
\end{tabular}


home programmes and client expectations. Clients perceived communication from students as inadequate. Clarity and understanding were factors deemed to be important in the writtten home exercise programme the students give the client to continue with on their own. There was an expectation that the students would provide medication. Clients also expected to improve after treatment, seeing the attention by students as a source of hope and motivation. Clients wanted to know what to expect from the students, particulary with regard to regular treatment. More frequent interventions were also desired.

\section{Student perspectives}

Data obtained from student interviews produced three main themes: differences, preparation for HBR, and learning. Differences noted in socioeconomic, cultural and language domains were repeatedly mentioned in the student interviews (Table 3). Although the students experienced culture and language as difficult areas for them, clients did not mention these issues. Differences between providing interventions in formal and informal treatment contexts were also frequently mentioned. There was a realisation that the usual physiotherapeutic interventions, which they would provide in a formal setting, were not necessarily appropriate in the home environment. The experience of seeing how clients function in their own environment was regarded as important in understanding the meaning of holistic intervention. They realised that there is more to treatment than merely the physical techniques they employ.

The theme of preparation for HBR was divided into the preparation the students required from the Division of Physiotherapy and the advice they felt would be helpful to give to future students (Table 4). The categories were subdivided to facilitate understanding of the data. The students thought that earlier exposure to resourceconstrained settings would be beneficial preparation for HBR. They pointed out, however, that a PowerPoint presentation at the beginning of the 4th year was not helpful. All students interviewed desired more language competency in isiXhosa. Handover of clients to new students was suggested to help to prepare them for what to expect and to assist with logistics and strategies for overcoming barriers.

Apart from preparation by the Division, the students had plenty of advice to give to future students, particularly with regard to setting

Table 2. Client-centredness

\begin{tabular}{ll}
\hline Category & Supporting quotations \\
\hline Communication & 'I wondered when they were coming back, or are they going away for good, so \\
there was no communication.' (CL5) & 'I was surprised that they didn't come back again. I didn't know what happened.' (CL3) \\
& 'How did they know about me?' (CL3) \\
Home programme & 'The student that drew the pictures really helped me a lot.' (CL2) \\
& 'The student made sure to show me until I understood.' (CL6) \\
Expectations of & 'I thought] they would come with tablets or something.' (CL3) \\
physiotherapy & 'The experience of standing up with them makes me believe I can walk again.' (CL7) \\
& 'I'm happy to see them because I want to be better.' (CL3) \\
Frequency of & 'If people come back, to know when and how many times.' (CL3) \\
treatment & 'Please come twice a week.' (CL7)
\end{tabular}

Table 3. Differences noted by students

\begin{tabular}{|c|c|}
\hline Category & Supporting quotations \\
\hline Location & $\begin{array}{l}\text { 'What could this patient's home environment actually be like, because sometimes you } \\
\text { can't even imagine.' (ST1) } \\
\text { 'You have to walk in between some interesting areas to get to your patient's house.' (ST6) }\end{array}$ \\
\hline Culture & $\begin{array}{l}\text { 'Completely different cultural setting, socioeconomic problems are completely different.' (ST1) } \\
\text { 'It gives you a culture shock; you do not expect what you see.' (ST6) }\end{array}$ \\
\hline $\begin{array}{l}\text { Informal } \\
\text { compared } \\
\text { with formal } \\
\text { treatment } \\
\text { settings }\end{array}$ & $\begin{array}{l}\text { 'Just because your patient can walk a little bit wobbly on tiles [in a clinic] doesn't mean } \\
\text { they are going to cope at home.' (ST6) } \\
\text { 'Techniques that we learn that would be good in the clinics, it literally does not work in } \\
\text { the community'. (ST4) } \\
\text { 'The success has almost got to do with more personal things than it has to do with } \\
\text { exactly what you're going to do.' (ST1) } \\
\text { 'It's actually more the talking, the social and psychological part that for them is more } \\
\text { important than the physical treatment.' (ST2) }\end{array}$ \\
\hline Language & $\begin{array}{l}\text { 'There was a serious communication barrier. Getting them to understand that you need } \\
\text { them to tell you what they're struggling with is a thing all on its own.' (ST6) } \\
\text { 'Even with my translator, it's difficult understanding them and getting my own point } \\
\text { across.' (ST3) }\end{array}$ \\
\hline
\end{tabular}

specific client-centred goals and being adaptable and organised (Table 5).

The last major theme emerging from the student data related to their learning (Table 6). Underlying the situated learning experience, various other learning theories were evident in students' comments. The need to adapt knowledge gained in the classroom and in other settings was sometimes a challenge in HBR. The students valued the learning opportunities afforded by working in pairs. The importance of learning from clients was also noted. Transformative learning experiences occurred from the experience of having to adapt to real-life situations, including in one instance dealing with the death of a client.

In summary, clients were grateful for the interventions received. There were, however, concerns regarding communication and unmet expectations. The students observed many differences between clients' socioeconomic contexts, culture and language, and their own. Differences in interventions in HBR compared with a formal setting were noted. Preparation desired in the curriculum included early exposure to communities and better language competency. Assistance was required with management and strategies for dealing with problems encountered. Preparation for HBR was preferred just before entering the community - not in advance.

\section{Discussion}

This article contributes to the understanding of physiotherapy students' and clients' experiences of HBR in an educational context. The need for improved client-centred communication was highlighted in both client and student data. 
Table 4. Preparation for home-based rehabilitation

\begin{tabular}{|c|c|c|}
\hline Category & Sub-category & Supporting quotations \\
\hline \multirow[t]{5}{*}{$\begin{array}{l}\text { Student suggestions } \\
\text { for preparation } \\
\text { by the Division of } \\
\text { Physiotherapy }\end{array}$} & Early exposure & $\begin{array}{l}\text { 'I don't know if this would be viable at all, but to almost have a } \\
\text { job-shadowing of a home visit [in 3rd year].' (ST1) } \\
\text { 'Having been exposed to it before you're very able to put the } \\
\text { new setting and culture shock at the back of your mind and get } \\
\text { on with why you were there.' (ST3) }\end{array}$ \\
\hline & Language & $\begin{array}{l}\text { 'Being able to speak Xhosa would've made a very big difference.' (ST6) } \\
\text { 'In 1st year, even though you're learning the words, you don't } \\
\text { realise why [isiXhosa is] so important. Once you see a patient } \\
\text { you understand. More exposure to Xhosa in our 3rd year } \\
\text { would also be best.' (ST1) }\end{array}$ \\
\hline & $\begin{array}{l}\text { Advance } \\
\text { preparation }\end{array}$ & $\begin{array}{l}\text { 'It's difficult to prepare someone a hundred percent for } \\
\text { something they've never seen before. A photo only says so much. } \\
\text { The actual area of the house is completely different.' (ST2) } \\
\text { '[A lecture early in year] you forget or don't really take it in } \\
\text { because it is so long until then.' (ST2) }\end{array}$ \\
\hline & Handover & $\begin{array}{l}\text { 'So that they know more or less what they can expect ... } \\
\text { they can be better prepared and know more than absolutely } \\
\text { nothing.' (ST6) } \\
\text { 'New students don't need to figure out all the barriers for } \\
\text { themselves from scratch.' (ST1) }\end{array}$ \\
\hline & Strategies & $\begin{array}{l}\text { 'Strategies to overcome the problem, because you see the } \\
\text { problem but you don't know [what to do].' (ST1) }\end{array}$ \\
\hline
\end{tabular}

Table 5. Preparation for home-based rehabilitation: Advice to future students

\begin{tabular}{|c|c|c|}
\hline Category & Sub-category & Supporting quotations \\
\hline \multirow[t]{8}{*}{$\begin{array}{l}\text { Advice to } \\
\text { future students }\end{array}$} & Professionalism & $\begin{array}{l}\text { 'Make yourself comfortable in someone else's house whether it's a } \\
\text { mansion or a shack. Be respectful of their environment.' (ST2) }\end{array}$ \\
\hline & Goal setting & 'Just being goal specific.' (ST4) \\
\hline & & 'Put your patient's needs first.' (ST3) \\
\hline & & $\begin{array}{l}\text { 'Do a really in-depth subjective [evaluation] and get to know them } \\
\text { and find out their goals etc.' (ST5) }\end{array}$ \\
\hline & Adaptability & $\begin{array}{l}\text { 'Don't be so eager to try to teach patients what you are taught } \\
\text { in class perfectly step by step; not be so technique driven in the } \\
\text { community because that's not going to get you anywhere, it doesn't } \\
\text { work.' (ST3) }\end{array}$ \\
\hline & & $\begin{array}{l}\text { 'The plan is never set in stone so don't forget that it should always } \\
\text { have room to be adapted.' (ST5) }\end{array}$ \\
\hline & Communication & $\begin{array}{l}\text { 'Discuss why we're only seeing you say once every two weeks.' } \\
\text { (ST1) }\end{array}$ \\
\hline & $\begin{array}{l}\text { Organisational } \\
\text { skills }\end{array}$ & $\begin{array}{l}\text { 'Just organising your patients better, making sure when to see who, } \\
\text { and making sure you have everything with you that you need.' (ST6) }\end{array}$ \\
\hline
\end{tabular}

Students identified early exposure to underresourced and culturally diverse settings as a prerequisite.

Clients' appreciation of HBR suggests that it is an important aspect of healthcare, providing treatment to clients who may otherwise not be able to access physiotherapeutic rehabilitation. However, clients seemed uncertain of the students' plans for their rehabilitation, particularly regard- ing the frequency of visits and termination of treatment, and were left wondering if the students would return. This suggests a lack of effective client-centred communication and planning. Stainsby and Bannigan ${ }^{[10]}$ regard the making of decisions on frequency and cessation of interventions as a skill; it appears that students need assistance with this aspect. The need for an in-depth and relevant subjective assessment to allow for appropriate collaborative goal setting with the client cannot be underestimated.

Mindful communication with clients and carers, reported by Tasker et al. ${ }^{[9]}$ is highlighted in the home environment to ensure relevant interventions. The differences in socioeconomic, racial, cultural and language backgrounds of these SU physiotherapy students compared with those of their clients may have decreased the efficacy of HBR. Communication and goal setting were most affected, as evidenced by students' comments on initial culture shock and the difficulty in client and therapist understanding each other. Development of the students' cultural competency and communication skills could enable the clients' understanding of the intervention and allow their desires to be heard. Reflection with peers and supervisors to address these issues should form an integral part of the placement. Although the clients did not comment that the students could not speak their language, this may have contributed to ineffective communication and management.

Language is an integral aspect of communication, and HBR highlights its significance in healthcare. ${ }^{[2,4,6]}$ The students stated that being proficient in isiXhosa would have helped in HBR to minimise the verbal communication barrier. They reported that an introductory isiXhosa course in their 1st year seemed irrelevant to them at the time. Once they started treating clients they realised the importance of learning the language. Therefore, agreeing with Prose et al. ${ }^{[8]}$ the possibility of a more timely course, which also promotes cultural competence, should be investigated. We agree with Mbalinda et al.$^{[6]}$ that a translator, who could also aid cultural understanding, can be a valuable communication tool. Although an interpreter accompanied the participants in this study on their home visits, there was still a barrier in communication with the client. This suggests that students should be taught how to facilitate better communication with clients through an interpreter or that a lack of language competency was not the only communication barrier.

Clients' lack of knowledge regarding physiotherapy may have contributed to communication barriers and unmet expectations, as seen in the common assumption that the students would provide medication. Therefore, client education is another aspect of client-centred communication. Another factor may be the rotation of different students through the placement, which may affect the consistency of treatment and progress 
Table 6. Learning facilitated by home-based rehabilitation

\begin{tabular}{ll}
\hline Category & Supporting quotations \\
\hline Authentic learning & 'In class you don't think of those kinds of things - an uneven path or it's steep.' (ST1) \\
& 'Think bigger with your treatment.' (ST3) \\
Social constructive & 'They always talk about tools in our toolkit, things that we've learnt and things \\
learning & that we can then apply to a patient, and that was sometimes a challenge.' (ST1) \\
& 'It's not outputs and techniques that we learn that would be good in the clinics. It \\
& literally does not work in the community. Use your initiative and be creative.' (ST3) \\
& 'Had to think out of the box a lot more. You learn to adapt - there were many life \\
& skills that you develop' (ST1) \\
Collaborative & 'We helped each other a lot and discussed situations.' (ST1) \\
learning & 'Your patients come up with the most interesting ways to do something.' (ST6) \\
Transformative & 'So you had to adapt to what the patient had. And it doesn't necessarily mean that your \\
learning & treatment is then poorer, it just means we had to think out of the box a lot more.'(ST1) \\
& 'My biggest lesson from those weeks spent in the community - you must remember \\
& where your patients are going once they leave you. It's made me treat my patients \\
& more holistically in an acute setting because I know some of the areas they are going \\
back to and that it's not ideal, you have to aim your treatment that way'. (ST3)
\end{tabular}

towards goals. As noted in a study on education in a community, collaboration with peers in the form of more comprehensive handover is necessary to build on what previous students achieved. ${ }^{[6]}$

Early exposure to the clinical environment as part of an integrated curriculum has been shown to increase student motivation and lead to deeper learning. ${ }^{[3,4]}$ Students suggested that earlier exposure may assist in overcoming the initial 'culture shock'. Experience of under-resourced environments and seeing clients in their own contexts will also help to situate physiotherapy practice from the beginning of the students' clinical training.

Providing HBR is complex, requiring the integration of many different skills. ${ }^{[4,9,10]}$ The students seemed to feel that they had adequate physiotherapy skills to conduct an intervention, but required greater adaptability in the HBR situation. A level of maturity is required to enable students to cope with the sometimes challenging real-life situations they experience. Therefore, HBR is better suited to the final year of study. However, facilitated exposure at the beginning of the clinical phase could be considered. In this study, students suggested that earlier exposure would assist in preparing them for the HBR context and help to minimise the reported culture shock. Collaborative learning from accompanying final-year students on home visits could be an option. Having a background of the clients' context may facilitate construction of more relevant knowledge at all the levels of care to which a student is exposed.

Students remarked on the differences between interventions in formal and informal settings. There is a paradigm shift in planning treatment from a purely physiotherapeutic approach to one that considers the client's context as paramount. This realisation of the need for a more holistic approach was also noted in other studies. ${ }^{[2,9]}$ Grappling with these adjustments leads to constructive and even transformative learning, as the students begin to think beyond the application of learned techniques to solving a client's problem. Students reported using their experience of the realities clients face to influence interventions in other contexts.

Students were silent on the issues of social accountability and responsibility, as well as the need for change in healthcare service delivery and their potential involvement in these areas. Although they were not specifically questioned on these aspects, it was hoped that a realisation of the place of HBR in the context of healthcare would emerge. This omission could indicate that clinical training in this context should specifically address the notion of social accountability. Students expressed a need for assistance in strategies to cope with the physical barriers they experienced in these settings. However, support is needed to assist them to recognise and deal with issues beyond the normal individualised intervention. Adopting a more reflective practice could, as part of this placement, facilitate transformative thinking, stimulating students to embrace the bigger picture and view their role as future agents of change. ${ }^{[2,3]}$

In summary, to ensure that students are able to gain full benefit from the exposure to HBR as a learning environment, they need effective and timely preparation just prior to entering the placement and continuous support to cope with the day-to-day challenges. Communication competence and specifically isiXhosa instruction are also needed. Facilitated early exposure to under-resourced communities should be considered. Effective preparation and support will assist the students to overcome the challenges of HBR and enhance the experience for clients.

This study cannot be generalised, as it focuses on the perspectives of a small sample in a specific setting. ${ }^{[14]}$ The use of research assistants may have limited the depth of probing during interviews; therefore, some comments may not have been explored sufficiently. Translation can result in some meaning being lost during interpretation.

Further investigation into the client and student experience in other community settings is required to achieve more in-depth information on the learning possibilities imbedded in the HBR experience. The effects of earlier exposure to underserved areas should be investigated in future to assess how this affects students' learning and practice. Follow-up of SU graduates to explore whether their HBR experience prepared them effectively for community service should be considered and the findings compared with those in previous similar studies. ${ }^{[2,4]}$

\section{Conclusion}

Exposure to real-life situations in underresourced settings in the form of HBR provides valuable situated and authentic learning opportunities for physiotherapy students. Clientcentredness, cultural competence, communication and adaptability are just some of the skills that students can develop and which will ultimately lead to enhanced client experiences. The HBR experience can be used to prepare graduates to address the needs of the populations they will serve during community service in SA.

Acknowledgements. This research has been supported by the President's Emergency Plan for AIDS Relief (PEPFAR) through Health Resources and Services Administration under the terms of T84HA21652, Stellenbosch University Rural Medical Education Partnership Initiative. 


\section{References}

1. Western Cape Government: Health. Healthcare 2030. The road to wellness. Draft. 2013. http:www.westerncape gov.za/health (accessed 7 May 2014)

2. Mostert-Wentzel K, Frantz J, van Rooijen AJ. A model for community physiotherapy from the perspective of newly graduated physiotherapists as a guide to curriculum revision. Afr J Health Professions Educ 2013;5(1):1925. [http://dx.doi.org/10.7196/AJHPE.203]

3. Boelen C, Woollard B. Social accountability and accreditation: A new frontier for educational institutions. Med Educ 2009;43:887-894. [http://dx.doi.org/10.1111/j.1365- 2923.2009.03413.x]

4. Ramklass S. Physiotherapists in under-resourced South African communities reflect on practice. Health Soc Care Community 2009;17(5):522-529. [http://dx.doi.org/10.1111/j.1365-2524.2009.00869.x]

5. Cameron D. Community-based education in a South African context: Was Socrates right? S Afr Fam Pract 2000;22(2):17-20.

6. Mbalinda S, Plover C, Burnham G, et al. Assessing community perspectives of the community-based education an service model at Makerere University, Uganda: A qualitative evaluation. Int Health Hum Rights 2011;11(Suppl 1):S6. 7. Chang W. Cultural competence of international humanitarian workers. Adult Educ Quart 2007;57(3):187-204. [http://dx.doi.org/10.1177/O741713606296755]
8. Prose N, Diab P, Matthews M. Experiential learning outside the comfort zone: Taking medical students to downtow Durban, South Africa. Afr J Health Professions Educ 2013;5(2):98-99. [http://dx.doi.org/10.7196/AJHPE.256] 9. Tasker D, Loftus S, Higgs, J. Head, heart and hands: Creating mindful dialogues in community-based physiotherapy. N Z J Physiother 2012;40(1):5-12.

10. Stainsby K, Bannigan K. Reviewing work-based learning opportunities in the community for physiotherapy students: An action research study. J Further and Higher Educ 2012;36(4):459-476. [http://dx.doi.org/10.1080/0309877X.2011.643769] 11. Mann K. Theoretical perspectives in medical education: Past experience and future possibilities. Med Educ 2011;45:60-68. [http://dx.doi.org/10.1111/j.1365-2923.2010.03757.x]

12. Van Schalkwyk S, Bezuidenhout J, Burch V, et al. Developing an educational research framework for evaluating rural training of health professionals: A case for innovation. Med Teach 2012;34(12):1064-1069. [http://dx.doi.org/10.3109/0 142159X.2012.719652]

3. Somekh B, Lewin C. Research Methods Social Sciences. London: Sage, 2005.

14. Silverman D, ed. Qualitative Research. London: Sage, 2011.

15. Du Plesis J He. Q

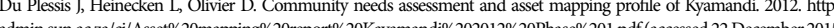
admin.sun.ac.za/ci/Asset\%20mapping\%20report\%20Kayamand1\%202012\%20Phase\%201.pdf (accessed 22 December 2015). 16. Frambach J, van der Vleuten C, Durning S. Quality criteria in qualitative and quantitative research. Acad Med 2013;88(4):552 